Research Article

\title{
Control of Hopf Bifurcation Type of a Neuron Model Using Washout Filter
}

\author{
Chunhua Yuan and Xiangyu Li $(D)$ \\ School of Automation and Electrical Engineering, Shenyang Ligong University, Shenyang 110159, China \\ Correspondence should be addressed to Xiangyu Li; xyli@sylu.edu.cn
}

Received 24 March 2021; Accepted 28 June 2021; Published 7 July 2021

Academic Editor: Massimiliano Ferrara

Copyright (c) 2021 Chunhua Yuan and Xiangyu Li. This is an open access article distributed under the Creative Commons Attribution License, which permits unrestricted use, distribution, and reproduction in any medium, provided the original work is properly cited.

\begin{abstract}
A quantitative mathematical model of neurons should not only include enough details to consider the dynamics of single neurons but also minimize the complexity of the model so that the model calculation is convenient. The two-dimensional Prescott model provides a good compromise between the authenticity and computational efficiency of a neuron. The dynamic characteristics of the Prescott model under external electrical stimulation are studied by combining analytical and numerical methods in this paper. Through the analysis of the equilibrium point distribution, the influence of model parameters and external stimulus on the dynamic characteristics is described. The occurrence conditions and the type of Hopf bifurcation in the Prescott model are analyzed, and the analytical determination formula of the Hopf bifurcation type in the neuron model is obtained. Washout filter control is used to change the Hopf bifurcation type, so that the subcritical Hopf bifurcation transforms to supercritical Hopf bifurcation, so as to realize the change of the dynamic characteristics of the model.
\end{abstract}

\section{Introduction}

Computational neuroscience emphasizes quantitative research methods and studies the nervous system at different levels through mathematical analysis and computer simulation [1]. Neuron is the smallest unit of the nervous system, and its structure and properties determine the functional characteristics of the neuron network [2]. Therefore, only by understanding the characteristics and activity of single neurons can we further understand the mystery of neuron networks and even the operation of the brain. For quantitative neuron models, it is necessary not only to include enough details to consider the dynamics of a single neuron but also to minimize the complexity of the model and retain its essential characteristics so that the model calculation is convenient [3]. The two need to reach a balance.

The single-compartment neuron models provide a good compromise between model authenticity and computational efficiency [4]. They ignore the spatial structure of neurons and highlight important features of neurons. They ignore unimportant details and only consider the specific ion channels or approximate morphological features that lead neurons to generate firing behaviors. They help us understand many dynamic phenomena of neurons, such as spike firing [5], cluster firing [6], and firing frequency adaptability [7]. Two-dimensional differential equations can be studied intuitively and visually through the method of phase plane analysis, which can not only reproduce rich discharge modes but also meet the nonlinear characteristics of studying dynamic behavior [8].

Neurons can be divided into two types according to excitability. Type I excitatory neurons can produce arbitrary low-frequency firing sequences under different intensities of external stimulation, while type II excitatory neurons cannot produce arbitrary low-frequency firing sequences. Repetitive firing can only be produced when the intensity of stimulation reaches the critical value [9]. Some models can only reproduce the characteristics of type I neurons (such as Hodgkin-Huxley model [10]), and others can only reproduce the characteristics of type II neurons (such as Morris-Lecar model [11]). However, in the Prescott model, these two types of excitability can be reproduced by changing key 
parameters. Therefore, the research in this paper is based on the two-dimensional Prescott neuron model.

A neuron is a dynamic system [12]. And phase trajectory is a common method to study the dynamic system. Changing the amplitude of the external stimulus will change the phase trajectory and firing state of a neuron. The qualitative change of the phase trajectory of the system is due to the bifurcation process of neuron dynamics [13]. For example, the type of bifurcation determines the excitability of neurons [14]. The subcritical Hopf bifurcation is an unstable limit cycle shrinking to a stable equilibrium point and making it out of equilibrium. And the phase trajectory becomes a large value limit cycle attractor. Supercritical Hopf bifurcation is a stable equilibrium point that loses stability and produces a limit cycle attractor with a small amplitude. With the increase in bifurcation parameters, the amplitude of the limit cycle gradually increases. Wang et al. explored the bifurcation mechanisms related to four types of bursters through the analysis of phase plane and calculated the first Lyapunov coefficient of the Hopf bifurcation, which can decide whether it is supercritical or subcritical [15]. Shi et al. proposed sufficient conditions for ensuring the system stability and derived relevant requirements for the generation of Hopf bifurcation with the help of the associated characteristic equation of the mathematical model [16].
Zhou et al. studied the local dynamic behaviors including stability and Hopf bifurcation of a four-dimensional hyperchaotic system with both analytical and numerical methods [17]. Bao et al. investigated the stability transitions of the stable and unstable equilibrium states via fold and Hopf bifurcations in a two-dimensional nonautonomous tabu learning neuron model [18]. However, few researchers have studied controlling of the bifurcation type of the twodimensional Prescott model by means of Washout filter.

In summary, this paper uses the theoretical method of nonlinear dynamics to study the bifurcation characteristics of the Prescott model. First, the equilibrium point distribution and the occurrence conditions for Hopf bifurcation are analyzed. Then, the analytical determination formula for the Hopf bifurcation type is deduced, and the bifurcation type of the Prescott model is judged. Finally, the bifurcation characteristics of the model are controlled using Washout filter.

\section{The Prescott Neuron Model and Its Equilibrium Points}

Prescott et al. proposed a two-dimensional neuron model consisting of a fast variable $V$ and a slow recovery variable $w$ in 2008 [9]. The dynamic equations are as follows:

$$
\begin{aligned}
c_{m} \dot{V} & =I-g_{L}\left(V-E_{L}\right)-g_{\mathrm{Na}} m_{\infty}(V)\left(V-E_{\mathrm{Na}}\right)-g_{\mathrm{K}} w\left(V-E_{\mathrm{K}}\right) \\
\dot{w} & =\frac{\varphi_{w}\left(w_{\mathrm{\infty}}(V)-w\right)}{\tau_{w}(V)},
\end{aligned}
$$

where $V$ is the neuron cell membrane voltage, $w$ is the slow ion channel recovery variable, and $I$ is the external stimulating current. $g_{\mathrm{Na}}, g_{\mathrm{K}}$, and $g_{L}$ are the maximum conductance of sodium ion channel, the maximum conductance of potassium ion channel, and the leakage conductance, respectively. $E_{\mathrm{Na}}, E_{\mathrm{K}}$, and $E_{L}$ are the corresponding back EMF. $c_{m}$ is the cell membrane capacitance of neurons. $m_{\infty}(V)$ is the steady state value of the sodium channel activation variable, $w_{\infty}(V)$ is the steady state value of the potassium channel recovery variable, and $\tau_{w}(V)$ is the time constant of the recovery variable. They are all functions of the neuron membrane voltage as follows:

$$
\begin{gathered}
m_{\infty}(V)=0.5\left(1+\tanh \left(\frac{V-\beta_{m}}{\gamma_{m}}\right)\right) \\
w_{\infty}(V)=0.5\left(1+\tanh \left(\frac{V-\beta_{m}}{\gamma_{m}}\right)\right), \\
\tau_{w}(V)=\frac{1}{\cosh \left(\left(V-\beta_{w}\right) / 2 \gamma_{w}\right)}
\end{gathered}
$$

where $\beta_{m}$ and $\gamma_{m}$ are the influencing factors of fast ion channel activation variable and $\beta_{w}$ and $\gamma_{w}$ are the influencing factors of slow ion channel recovery variable. Among them, $\beta_{w}$ is the key parameter of the model because changing $\beta_{w}$ can simulate various firing patterns of the model.

The values of the model parameters in this paper are as follows: $c_{m}=2 \mu \mathrm{F} / \mathrm{cm}^{2}, \varphi_{w}=0.15, g_{L}=2 \mathrm{mS} / \mathrm{cm}^{2}, g_{\mathrm{Na}}=$ $20 \mathrm{mS} / \mathrm{cm}^{2}, g_{k}=20 \mathrm{mS} / \mathrm{cm}^{2}, E_{L}=-70 \mathrm{mV}, E_{\mathrm{Na}}=50 \mathrm{mV}$, $E_{\mathrm{K}}=-100 \mathrm{mV}, \beta_{m}=-1.2 \mathrm{mV}, \gamma_{m}=18 \mathrm{mV}, \beta_{w}=-10 \mathrm{mV}$, and $\gamma_{w}=10 \mathrm{mV}$.

The different firing characteristics of neurons are related to the type and stability of the equilibrium point of the model. The type of equilibrium point can be judged by the eigenvalues of the model equation. Let

$$
\begin{aligned}
& f_{1}=\frac{\left[I-g_{L}\left(V-E_{L}\right)-g_{\mathrm{Na}} m_{\infty}(V)\left(V-E_{\mathrm{Na}}\right)-g_{\mathrm{K}} w\left(V-E_{\mathrm{K}}\right)\right]}{c_{m}}, \\
& f_{2}=\frac{\varphi_{w}\left(w_{\infty}(V)-w\right)}{\tau_{w}(V)} .
\end{aligned}
$$

Then, the eigenvalue $\lambda$ of the neuron dynamic equations can be obtained from $|\mathbf{A}-\lambda \mathbf{I}|=0$ and written as a polynomial of $\lambda^{2}+p \lambda+q=0$, where $\mathbf{A}$ is the linearized matrix at the equilibrium point of the equation and $\mathbf{I}$ is the identity matrix: 


$$
\mathbf{A}=\left[\begin{array}{ll}
a & b \\
c & d
\end{array}\right]=\left[\begin{array}{ll}
\frac{\partial f_{1}}{\partial V} & \frac{\partial f_{1}}{\partial w} \\
\frac{\partial f_{2}}{\partial V} & \frac{\partial f_{2}}{\partial w}
\end{array}\right]_{\substack{V=V_{0} \\
w=w_{0}}},
$$

where

$$
\begin{aligned}
& \frac{\partial f_{1}}{\partial V}=\frac{1}{c_{m}}\left[-g_{L}-\frac{g_{\mathrm{Na}}}{2 \gamma_{m} \cosh ^{2}\left(\left(V-\beta_{m}\right) / \gamma_{m}\right)}\left(V-E_{\mathrm{Na}}\right)-\frac{g_{\mathrm{Na}}}{2}\left(1+\tanh \left(\frac{V-\beta_{m}}{\gamma_{m}}\right)\right)-g_{\mathrm{K}} w\right], \\
& \frac{\partial f_{1}}{\partial w}=\frac{\left[-g_{K}\left(V-E_{\mathrm{K}}\right)\right]}{c_{m}}, \\
& \frac{\partial f_{2}}{\partial V}=\frac{\varphi_{w}}{2 \gamma_{w}} \frac{\cosh \left(\left(V-\beta_{w}\right) / 2 \gamma_{w}\right)}{\cosh ^{2}\left(\left(V-\beta_{w}\right) / \gamma_{w}\right)}+\varphi_{w}\left[0.5\left(1+\tanh \left(\frac{V-\beta_{w}}{\gamma_{w}}\right)\right)-w\right] \frac{\sinh \left(\left(V-\beta_{w}\right) / 2 \gamma_{w}\right)}{2 \gamma_{w}}, \\
& \frac{\partial f_{2}}{\partial w}=-\varphi_{w} \cosh \left(\frac{V-\beta_{w}}{2 \gamma_{w}}\right) .
\end{aligned}
$$

The two solutions of the polynomial $\lambda^{2}+p \lambda+q=0$ are the eigenvalues of the characteristic equation as follows:

$$
\lambda_{1,2}=\frac{1}{2}\left[-p \pm \sqrt{p^{2}-4 q}\right]
$$

where $p=-(a+d)$ and $q=a d-b c$.

According to the eigenvalues of the equilibrium point of the model, the type of the equilibrium point can be judged and the influence on the firing behavior of neurons can be further analyzed.

Figure 1 shows the equilibrium bifurcation diagram of the Prescott model, in which the pink circle, the black dot, the blue intersection, and the black cross represent the unstable focus, the stable focus, the saddle, and the stable node, respectively. When the stimulation current is between $I=32.78\left(\mu \mathrm{A} / \mathrm{cm}^{2}\right) \sim 33.2\left(\mu \mathrm{A} / \mathrm{cm}^{2}\right)$, the model has three equilibrium points.

\section{Hopf Bifurcation Analysis of the Prescott Model}

When a certain parameter in the system changes, the stability of the equilibrium point of the nonlinear system changes. The stable focus turns into an unstable focus and a limit cycle is generated near it, and the Hopf bifurcation phenomenon occurs. At this time, the equilibrium point becomes the center point, and its eigenvalues become pure imaginary number. When the bifurcation parameter changes to the bifurcation value, the unstable limit cycle shrinks to a stable equilibrium point and turns it into an unstable equilibrium point.

It can be seen from the above that the conditions for the occurrence of Hopf bifurcation in a two-dimensional nonlinear system are (1) $p=0$ and (2) $q>0$. The eigenvalues are pure imaginary numbers at this time, and the equilibrium point is the center point.

For the Prescott model,

(1) $p=0$ : Because $p=-(a+d)$, it can be seen from formula (5):

$$
p=\frac{\left[g_{L}+g_{\mathrm{Na}} m_{\infty}^{\prime}(V)\left(V-E_{\mathrm{Na}}\right)+g_{\mathrm{Na}} m_{\infty}(V)+g_{\mathrm{K}} w\right]}{c_{m}}+\varphi_{w} \cosh \left(\frac{V-\beta_{w}}{2 \gamma_{w}}\right)=0 .
$$

Therefore,

$$
w=\frac{\left[-c_{m} \varphi_{w} \cosh \left(\left(V-\beta_{w}\right) / 2 \gamma_{w}\right)-g_{L}-g_{\mathrm{Na}} m_{\infty}^{\prime}(V)\left(V-E_{\mathrm{Na}}\right)-g_{\mathrm{Na}} m_{\infty}(V)\right]}{g_{\mathrm{K}}} .
$$




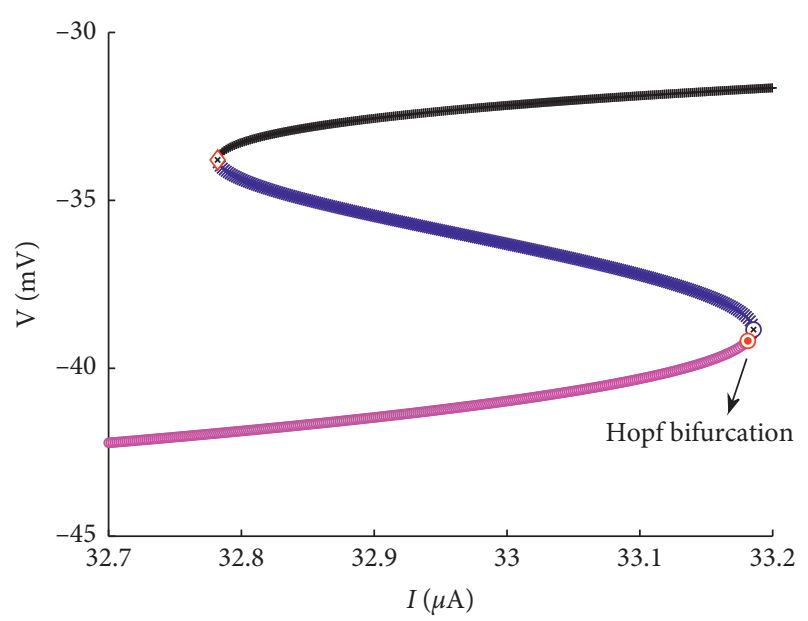

FIGURE 1: Distribution of equilibrium points of the Prescott model.

If the equilibrium point $\left(V_{0}, w_{0}\right)$ of the model satisfies formula (8), then the coefficient of the firstorder term of the characteristic equation of the equilibrium point is zero.
(2) $q>0$

According to condition (1), $p=a+d=0$, so $q=a d-b c=-a^{2}-b c>0$, that is, $a^{2}+b c<0$. Therefore, this condition is equivalent to (1) $b c<0$; that is, $b$ and $c$ are opposite signs and (2) $|b c|>a^{2}$ or $|b c|>d^{2}$.

From formula (5), $b=\left[-g_{\mathrm{K}}\left(V-E_{\mathrm{K}}\right)\right] / c_{m}$. For the Prescott model, $E_{\mathrm{K}}=-100 \mathrm{mV}$, and usually the membrane voltage will not be less than $-100 \mathrm{mV}$, so $V>E_{K}$. At this time, if $V>E_{K}$ is required, only let $b c<0$, that is,

$$
c=\frac{\varphi_{w}\left[w_{\infty}^{\prime}(V) \tau_{w}(V)-\left(w_{\infty}(V)-w\right) \tau_{w}^{\prime}(V)\right]}{\left[\tau_{w}(V)\right]^{2}}>0 .
$$
thus,

When the neuron model is at equilibrium, $w=w_{\infty}(V)$;

$$
\varphi_{w} w_{\infty}^{\prime}(V) \tau_{w}(V)>0 .
$$

Substituting $w_{\infty}^{\prime}(V)=\left(0.5 / \gamma_{w}\right)\left(1 / \cosh ^{2}\left(\left(V-\beta_{w}\right) / \gamma_{w}\right)\right)$ and $\tau_{w}(V)=1 / \cosh \left(\left(V-\beta_{w}\right) / 2 \gamma_{w}\right)$, we get

$$
\frac{\varphi_{w}}{2 \gamma_{w}} \frac{1}{\cosh ^{2}\left(\left(V-\beta_{w}\right) / \gamma_{w}\right) \cdot \cosh \left(\left(V-\beta_{w}\right) / 2 \gamma_{w}\right)} \text { is always greater than zero. }
$$

That is, when the neuron model is under given parameters and the coefficient of its characteristic equation $p=0$, condition (1) is automatically satisfied.

Condition (2): $|b c|>d^{2}$, that is,

$$
\left|\frac{-g_{\mathrm{K}}\left(V-E_{\mathrm{K}}\right) \varphi_{w} w_{\infty}^{\prime}(V)}{c_{m} \tau_{w}(V)}\right|>\varphi_{w}^{2} \cosh ^{2}\left(\frac{V-\beta_{w}}{2 \gamma_{w}}\right) .
$$

The hyperbolic cosine function is a constant positive function, so we can get

$$
\left|\frac{-g_{\mathrm{K}}\left(V-E_{\mathrm{K}}\right)}{2 c_{m} \gamma_{w} \varphi_{w} \cosh ^{2}\left(\left(V-\beta_{w}\right) / \gamma_{w}\right) \cosh \left(\left(V-\beta_{w}\right) / 2 \gamma_{w}\right)}\right|>1 .
$$

Therefore, when the equilibrium point of the Prescott model satisfies both formulae (8) and (13), Hopf bifurcation occurs in the Prescott model.

The local magnification near the Hopf bifurcation of the Prescott model is shown in Figure 2, and there is a very small range of stable focus between the unstable focuses and the saddles. When $I=33.1813 \mu \mathrm{A} / \mathrm{cm}^{2}$, the Hopf bifurcation occurs in the model, and the membrane voltage is $V=-39.1846 \mathrm{mV}$ at this time.

\section{The Type of Hopf Bifurcation in the Prescott Model}

Suppose the nonlinear system equation of the two-dimensional Prescott model is

$$
\dot{x}=\mathbf{f}(\mathbf{x}, \mu),
$$

where $\mathbf{x}=\left[x_{1}, x_{2}\right]^{T}$ and $\mathbf{f}=\left[f_{1}, f_{2}\right]^{T}$. The equilibrium point $\mathbf{x}_{0}\left(\mu_{0}\right)=\left[x_{10}\left(\mu_{0}\right), x_{20}\left(\mu_{0}\right)\right]^{T}$ is obtained from the equilibrium equation $\dot{x}=\mathbf{f}(\mathbf{x}, \mu)=0$. And the linear part of the model is extracted and rewritten as follows:

$$
\dot{x}=\mathbf{f}(\mathbf{x}, \mu)=\mathbf{A} \mathbf{x}+\mathbf{g}(\mathbf{x})
$$

where $\mathbf{A}$ is the Jacobian matrix at the equilibrium point and $\mathbf{g}(\mathbf{x})$ is the nonlinear term as follows:

$$
\begin{aligned}
& \mathbf{A}=\left.\frac{\partial \mathbf{f}}{\partial \mathbf{x}}\right|_{\mathbf{x}=\mathbf{x}_{0}}=\left[\begin{array}{ll}
\frac{\partial f_{1}}{\partial x_{1}} & \frac{\partial f_{1}}{\partial x_{2}} \\
\frac{\partial f_{2}}{\partial x_{1}} & \frac{\partial f_{2}}{\partial x_{2}}
\end{array}\right]_{\substack{x_{1}=x_{10} \\
x_{2}=x_{20}}}, \\
& \mathbf{g}(x)=\mathbf{f}(\mathbf{x}, \mu)-\mathbf{A x} .
\end{aligned}
$$

A linear transformation is performed on the two-dimensional system: $\mathbf{x}=\mathbf{P y}+\mathbf{x}_{0}\left(\mu_{0}\right)$, where $\mathbf{P}=$ $\left[\operatorname{Re}\left(\lambda_{1}\right) \operatorname{Im}\left(\lambda_{1}\right)\right]$ is a two-dimensional real number matrix composed of the real and imaginary parts of the eigenvector corresponding to the eigenvalue $\lambda_{1}=i \omega_{0}(\mu)$ of the Jacobian matrix A. So,

$$
\dot{x}=\mathbf{P} \dot{y}=\mathbf{A}\left(\mathbf{P y}+\mathbf{x}_{0}\left(\mu_{0}\right)\right)+\mathbf{g}\left(\mathbf{P} \mathbf{y}+\mathbf{x}_{0}\left(\mu_{0}\right)\right) .
$$

Substituting formulae (2)-(27) into (2)-(28), we get 


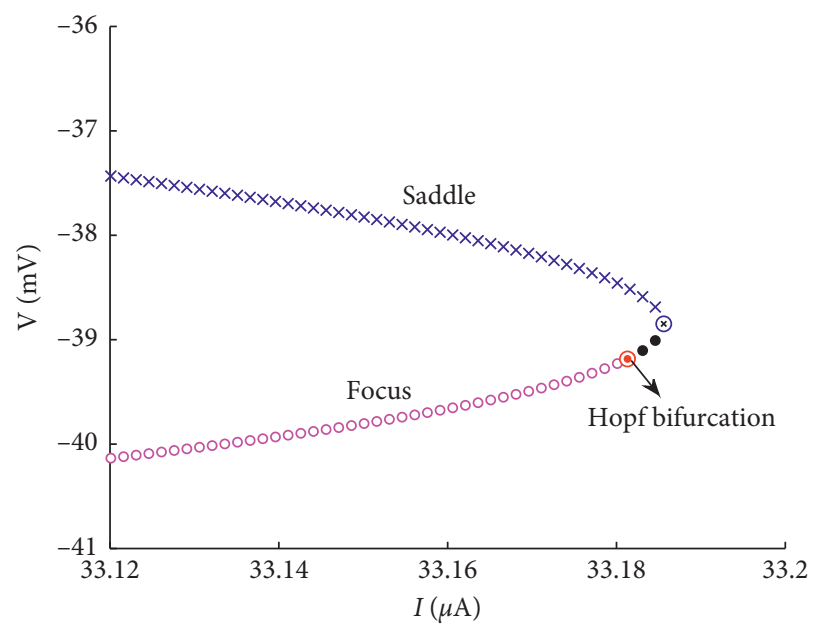

FIgURe 2: Local magnifications of equilibrium point distribution near the Hopf bifurcation of the Prescott model.

$\mathbf{P} \dot{y}=\mathbf{A}\left(\mathbf{P y}+\mathbf{x}_{0}\left(\mu_{0}\right)\right)+\mathbf{f}\left(\mathbf{P y}+\mathbf{x}_{0}\left(\mu_{0}\right)\right)-\mathbf{A}\left(\mathbf{P y}+\mathbf{x}_{0}\left(\mu_{0}\right)\right) . \quad$ Therefore

$$
\begin{aligned}
\dot{y} & =\mathbf{P}^{-1} \mathbf{A}\left(\mathbf{P y}+\mathbf{x}_{0}\left(\mu_{0}\right)\right)+\mathbf{P}^{-1}\left[\mathbf{f}\left(\mathbf{P y}+\mathbf{x}_{0}\left(\mu_{0}\right)\right)-\mathbf{A}\left(\mathbf{P y}+\mathbf{x}_{0}\left(\mu_{0}\right)\right)\right] \\
& =\mathbf{P}^{-1} \mathbf{A} \mathbf{P} \mathbf{y}+\mathbf{P}^{-1} \mathbf{f}\left(\mathbf{P y}+\mathbf{x}_{0}\left(\mu_{0}\right)\right)-\mathbf{P}^{-1} \mathbf{A P y} \\
& =\mathbf{J y}+\mathbf{P}^{-1} \mathbf{f}\left(\mathbf{P y}+\mathbf{x}_{0}\left(\mu_{0}\right)\right)-\mathbf{J y} \\
& =\mathbf{J y}+\mathbf{F},
\end{aligned}
$$

where $\mathbf{J}=\mathbf{P}^{-1} \mathbf{A P}$ is the Jacobian matrix of the system and $\mathbf{F}$ is the nonlinear term as follows:

$$
\mathbf{F}=\mathbf{P}^{-1} \mathbf{f}\left(\mathbf{P y}+\mathbf{x}_{0}\left(\mu_{0}\right)\right)-\mathbf{J y} \text {. }
$$

Its component form is

$$
\left[\begin{array}{c}
F_{1} \\
F_{2}
\end{array}\right]=\mathbf{P}^{-1}\left[\begin{array}{l}
f_{1}\left(\mathbf{P y}+\mathbf{x}_{0}\left(\mu_{0}\right)\right) \\
f_{2}\left(\mathbf{P y}+\mathbf{x}_{0}\left(\mu_{0}\right)\right)
\end{array}\right]-\mathbf{J}\left[\begin{array}{l}
y_{1} \\
y_{2}
\end{array}\right] .
$$

On the basis of the Hopf bifurcation theory [19], the determination formula of Hopf bifurcation type is derived as follows:

$$
\beta_{2}=2 \operatorname{Re}\left\{\frac{g_{20} g_{11}-2\left|g_{11}\right|^{2}-\left(1 / 3\left|g_{02}\right|^{2}\right)}{2 \omega_{0}} i+\frac{g_{21}}{2}\right\} .
$$

It can be simplified to

$$
\beta_{2}=\operatorname{Re}\left\{\frac{g_{20} g_{11}}{\omega_{0}} i+g_{21}\right\}
$$

where

$$
\begin{aligned}
& g_{20}=\frac{1}{4}\left(\frac{\partial^{2} F_{1}}{\partial y_{1}^{2}}-\frac{\partial^{2} F_{1}}{\partial y_{2}^{2}}+2 \frac{\partial^{2} F_{2}}{\partial y_{1} \partial y_{2}}+i\left(\frac{\partial^{2} F_{2}}{\partial y_{1}^{2}}-\frac{\partial^{2} F_{2}}{\partial y_{2}^{2}}-2 \frac{\partial^{2} F_{1}}{\partial y_{1} \partial y_{2}}\right)\right) \\
& g_{11}=\frac{1}{4}\left(\frac{\partial^{2} F_{1}}{\partial y_{1}^{2}}+\frac{\partial^{2} F_{1}}{\partial y_{2}^{2}}+i\left(\frac{\partial^{2} F_{2}}{\partial y_{1}^{2}}+\frac{\partial^{2} F_{2}}{\partial y_{2}^{2}}\right)\right), \\
& g_{21}=G_{21}+\sum_{k=1}^{n-2}\left(2 G_{110}^{k} w_{11}^{k}+G_{101}^{k} w_{20}^{k}\right) .
\end{aligned}
$$


For two-dimensional nonlinear systems, $n=2$; thus, $g_{21}=G_{21}$; therefore,

$$
\begin{aligned}
\beta_{2}= & -\frac{1}{16 \omega_{0}}\left[\left(\frac{\partial^{2} F_{1}}{\partial y_{1}^{2}}-\frac{\partial^{2} F_{1}}{\partial y_{2}^{2}}+2 \frac{\partial^{2} F_{2}}{\partial y_{1} \partial y_{2}}\right)\left(\frac{\partial^{2} F_{2}}{\partial y_{1}^{2}}+\frac{\partial^{2} F_{2}}{\partial y_{2}^{2}}\right)+\left(\frac{\partial^{2} F_{2}}{\partial y_{1}^{2}}-\frac{\partial^{2} F_{2}}{\partial y_{2}^{2}}-2 \frac{\partial^{2} F_{1}}{\partial y_{1} \partial y_{2}}\right)\left(\frac{\partial^{2} F_{1}}{\partial y_{1}^{2}}+\frac{\partial^{2} F_{1}}{\partial y_{2}^{2}}\right)\right] \\
& +\frac{1}{8}\left(\frac{\partial^{3} F_{1}}{\partial y_{1}^{3}}+\frac{\partial^{3} F_{1}}{\partial y_{1} \partial y_{2}^{2}}+\frac{\partial^{3} F_{2}}{\partial y_{1}^{2} \partial y_{2}}+\frac{\partial^{3} F_{2}}{\partial y_{2}^{3}}\right) .
\end{aligned}
$$

That is,

$$
\begin{aligned}
\beta_{2}= & \frac{1}{8 \omega_{0}}\left[\frac{\partial^{2} F_{1}}{\partial y_{2}^{2}} \cdot \frac{\partial^{2} F_{2}}{\partial y_{2}^{2}}-\frac{\partial^{2} F_{1}}{\partial y_{1}^{2}} \cdot \frac{\partial^{2} F_{2}}{\partial y_{1}^{2}}+\frac{\partial^{2} F_{1}}{\partial y_{1} \cdot \partial y_{2}} \cdot\left(\frac{\partial^{2} F_{1}}{\partial y_{1}^{2}}+\frac{\partial^{2} F_{1}}{\partial y_{2}^{2}}\right)-\frac{\partial^{2} F_{2}}{\partial y_{1} \cdot \partial y_{2}}\left(\frac{\partial^{2} F_{2}}{\partial y_{1}^{2}}+\frac{\partial^{2} F_{2}}{\partial y_{2}^{2}}\right)\right] \\
& +\frac{1}{8}\left[\frac{\partial^{3} F_{2}}{\partial y_{2}^{3}}+\frac{\partial^{3} F_{1}}{\partial y_{1} \cdot \partial y_{2}^{2}}+\frac{\partial^{3} F_{2}}{\partial y_{1}^{2} \cdot \partial y_{2}}+\frac{\partial^{3} F_{1}}{\partial y_{1}^{3}}\right],
\end{aligned}
$$

where $\omega_{0}$ is the imaginary part of the pure imaginary eigenvalues of the Jacobian matrix of the two-dimensional system and is greater than zero.

When $\beta_{2}>0$, the subcritical Hopf bifurcation occurs in the system, and the new equilibrium state branch is unstable when the bifurcation parameter is greater than the bifurcation value. When $\beta_{2}<0$, the system undergoes a supercritical Hopf bifurcation. When the bifurcation parameter changes to the bifurcation value, the corresponding equilibrium point becomes an unstable equilibrium point and a stable limit cycle is generated.

From formulae (1) and (21), the nonlinear term of the Prescott model equation can be derived as follows:

$$
\begin{aligned}
f_{1}= & \frac{1}{c_{m}}\left[I-g_{L}\left(-E_{L}+x_{10}+P_{11} \cdot y_{1}+P_{12} \cdot y_{2}\right)-g_{\mathrm{K}}\left(-E_{\mathrm{K}}+x_{10}+P_{11} \cdot y_{1}+P_{12} \cdot y_{2}\right)\left(x_{20}+P_{21} \cdot y_{1}+P_{22} \cdot y_{2}\right)\right. \\
& \left.-0.5 g_{\mathrm{Na}}\left(-E_{\mathrm{Na}}+x_{10}+P_{11} \cdot y_{1}+P_{12} \cdot y_{2}\right)\left(1+\tanh \left(\frac{x_{10}+P_{11} \cdot y_{1}+P_{12} \cdot y_{2}-\beta_{m}}{\gamma_{m}}\right)\right)\right] \\
f_{2}= & \cosh \left(\frac{x_{10}+P_{11} \cdot y_{1}+P_{12} \cdot y_{2}-\beta_{w}}{2 \gamma_{w}}\right) \varphi_{w}\left[-\left(x_{20}+P_{21} \cdot y_{1}+P_{22} \cdot y_{2}\right)+0.5\left(1+\tanh \left(\frac{x_{10}+P_{11} \cdot y_{1}+P_{12} \cdot y_{2}-\beta_{w}}{\gamma_{w}}\right)\right)\right] .
\end{aligned}
$$

Since Jy is a first-order function of $\mathbf{y}$, while in the following calculations, at least the second derivative of $y$ is calculated. So, only $\mathbf{F}=\mathbf{P}^{-1} \mathbf{f}\left(\mathbf{P y}+\mathbf{x}_{0}\left(\mu_{0}\right)\right)$ is needed to be calculated as follows: 


$$
\begin{aligned}
& F_{1}=\frac{P_{22}}{c_{m}\left(P_{11} P_{22}-P_{12} P_{21}\right)}\left[I-g_{L}\left(-E_{L}+x_{10}+P_{11} \cdot y_{1}+P_{12} \cdot y_{2}\right)\right. \\
& -g_{\mathrm{K}}\left(-E_{\mathrm{K}}+x_{10}+P_{11} \cdot y_{1}+P_{12} \cdot y_{2}\right)\left(x_{20}+P_{21} \cdot y_{1}+P_{22} \cdot y_{2}\right) \\
& \left.-0.5 g_{\mathrm{Na}}\left(-E_{\mathrm{Na}}+x_{10}+P_{11} \cdot y_{1}+P_{12} \cdot y_{2}\right)\left(1+\tanh \left(\frac{x_{10}+P_{11} \cdot y_{1}+P_{12} \cdot y_{2}-\beta_{m}}{\gamma_{m}}\right)\right)\right] \\
& -\frac{P_{21}}{P_{11} P_{22}-P_{12} P_{21}} \cosh \left(\frac{x_{10}+P_{11} \cdot y_{1}+P_{12} \cdot y_{2}-\beta_{w}}{2 \gamma_{w}}\right) \varphi_{w} . \\
& {\left[-\left(x_{20}+P_{21} \cdot y_{1}+P_{22} \cdot y_{2}\right)+0.5\left(1+\tanh \left(\frac{x_{10}+P_{11} \cdot y_{1}+P_{12} \cdot y_{2}-\beta_{w}}{\gamma_{w}}\right)\right)\right],} \\
& F_{2}=\frac{-P_{12}}{c_{m}\left(P_{11} P_{22}-P_{12} P_{21}\right)}\left[I-g_{L}\left(-E_{L}+x_{10}+P_{11} \cdot y_{1}+P_{12} \cdot y_{2}\right)\right. \\
& -g_{K}\left(-E_{\mathrm{K}}+x_{10}+P_{11} \cdot y_{1}+P_{12} \cdot y_{2}\right)\left(x_{20}+P_{21} \cdot y_{1}+P_{22} \cdot y_{2}\right) \\
& \left.-0.5 g_{\mathrm{Na}}\left(-E_{\mathrm{Na}}+x_{10}+P_{11} \cdot y_{1}+P_{12} \cdot y_{2}\right) \cdot\left(1+\tanh \left(\frac{x_{10}+P_{11} \cdot y_{1}+P_{12} \cdot y_{2}-\beta_{m}}{\gamma_{m}}\right)\right)\right] \\
& +\frac{P_{11}}{P_{11} P_{22}-P_{12} P_{21}} \cosh \left(\frac{x_{10}+P_{11} \cdot y_{1}+P_{12} \cdot y_{2}-\beta_{w}}{2 \gamma_{w}}\right) \phi_{w} \\
& {\left[-\left(x_{20}+P_{21} \cdot y_{1}+P_{22} \cdot y_{2}\right)+0.5\left(1+\tanh \left(\frac{x_{10}+P_{11} \cdot y_{1}+P_{12} \cdot y_{2}-\beta_{w}}{\gamma_{w}}\right)\right)\right] .}
\end{aligned}
$$

The relevant derivatives of each nonlinear term, the parameters of the model, and the Hopf bifurcation parameter are substituted into formula (26) to calculate the bifurcation type determination formula $\beta_{2}$. If $\beta_{2}<0$, a supercritical Hopf bifurcation occurs at this equilibrium point; if $\beta_{2}>0$, a subcritical Hopf bifurcation occurs at this equilibrium point.

It is shown in Section 3 that when $I_{0}=33.1813 \mu \mathrm{A} / \mathrm{cm}^{2}$, the Prescott model has Hopf bifurcation at the equilibrium point $V_{0}=-39.1842 \mathrm{mV}$ and $w_{0}=0.002961$. The corresponding eigenvalues are $\lambda_{1}=0.06583 i$ and $\lambda_{2}=-0.06583 i$. So, the matrix $\mathbf{P}$ corresponding to the eigenvalue of the linearized matrix of the system here is as follows:

$$
\mathbf{P}=\left[\begin{array}{ll}
P_{11} & P_{12} \\
P_{21} & P_{22}
\end{array}\right]=\left[\begin{array}{cc}
0.99999984 & 0 \\
0.00055926742 & 0.00010825119
\end{array}\right] .
$$

Substituting the equilibrium point value $\left(V_{0}, w_{0}\right)$, the bifurcation parameter $I_{0}$, and the eigenvector $\mathbf{P}$ at the bifurcation into the bifurcation type determination formula (26), $\beta_{2}=0.05306736>0$ is obtained, indicating that the
Prescott model has a subcritical Hopf bifurcation at $\left(V_{0}, w_{0}\right)$, and the new equilibrium state branch is unstable.

The firing response curve of the Prescott model at $\left(V_{0}, w_{0}\right)=(-39.1842,0.002961)$ is shown in Figure 3. The model is disturbed by the external simulation, and the stable equilibrium point loses its stability, resulting in a large-scale limit cycle. At the same time, the system produces a largeamplitude oscillation.

\section{Hopf Bifurcation Type Control Based on Washout Filter}

The subcritical Hopf bifurcation will cause the new equilibrium state of the system to lose stability. In order to avoid this situation, this section designs a controller based on the Washout filter to control the subcritical Hopf bifurcation caused by the bifurcation parameter $I$. The Washout filter is selected because it does not change the equilibrium point position of the system after adding the controller so as not to 


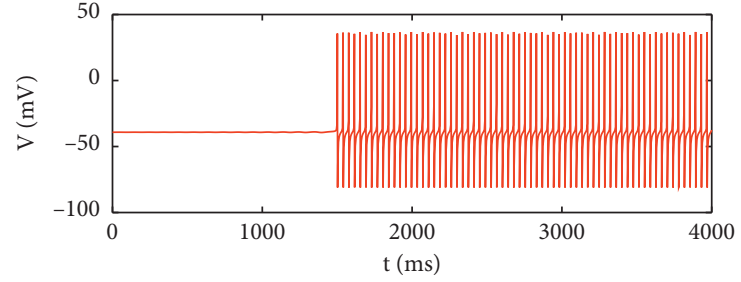

(a)

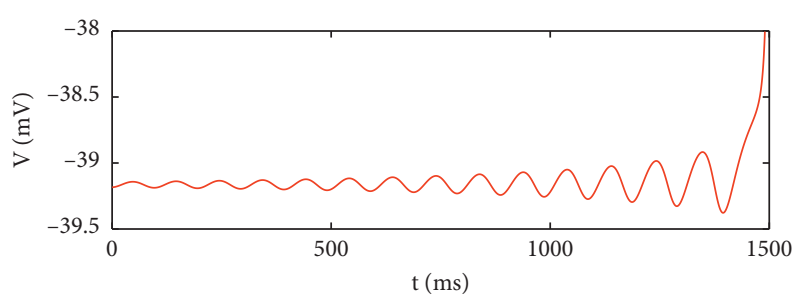

(b)

FIgURE 3: The firing response curve (a) and its local magnification (b) in the neighborhood of the subcritical Hopf bifurcation in the Prescott model.

change the operating state of the system and avoid the waste of energy. Therefore, the Washout filter is used to change the Hopf bifurcation type of the neuron model. The dynamic equations of the system after adding the controller are as follows:

$$
\begin{aligned}
c_{m} \dot{V}= & I-g_{L}\left(V-E_{L}\right)-g_{\mathrm{Na}} m_{\infty}(V)\left(V-E_{\mathrm{Na}}\right) \\
& -g_{\mathrm{K}} w\left(V-E_{E}\right)+u, \\
\dot{w}= & \frac{\varphi_{w}\left(w_{\infty}(V)-w\right)}{\tau(V)}, \\
\dot{z}= & V-\xi z, \\
u= & k(V-\xi z)^{3}, \\
m_{\infty}(V)= & 0.5\left(1+\tanh \left(\frac{V-\beta_{m}}{\gamma_{m}}\right)\right), \\
w_{\infty}(V)= & 0.5\left(1+\tanh \left(\frac{V-\beta_{w}}{\gamma_{w}}\right)\right), \\
\tau(V)= & \frac{\cosh \left(\left(V-\beta_{w}\right) / 2 \gamma_{w}\right)}{1}
\end{aligned}
$$

where $u$ is the Washout nonlinear controller, $z$ is the state variable of the controller, $k$ is the gain of the Washout filter, and $\xi$ is the reciprocal of the time constant of the filter, which is 0.5 in this paper.

The controlled Prescott model constitutes a three-dimensional nonlinear system, and its Hopf bifurcation stability needs to be determined according to the highdimensional Hopf bifurcation theory. Suppose the nonlinear equation of the three-dimensional system is as follows: $\dot{x}=\mathbf{f}(\mathbf{x}, \mu)$, where $\mathbf{x}=\left[x_{1}, x_{2}, x_{3}\right]^{T}$ and $\mathbf{f}=\left[f_{1}, f_{2}, f_{3}\right]^{T}$. Let $\dot{x}=\mathbf{f}(\mathbf{x}, \mu)=0$, find the equilibrium point $\mathbf{x}_{0}$ $\left(\mu_{0}\right)=\left[x_{10}\left(\mu_{0}\right), x_{20}\left(\mu_{0}\right), x_{30}\left(\mu_{0}\right)\right]^{T}$. Extract the linear part of the equation and rewrite it as $\dot{x}=\mathbf{f}(\mathbf{x}, \mu)=\mathbf{A x}+\mathbf{g}(\mathbf{x})$, where $\mathbf{A}$ is the Jacobian matrix at the equilibrium point and $\mathbf{g}(\mathbf{x})$ is the nonlinear term as follows:

$$
\begin{aligned}
\mathbf{A}=\left.\frac{\partial \mathbf{f}}{\partial \mathbf{x}}\right|_{\mathbf{x}=\mathbf{x}_{0}}=\left[\begin{array}{lll}
\frac{\partial f_{1}}{\partial x_{1}} & \frac{\partial f_{1}}{\partial x_{2}} & \frac{\partial f_{1}}{\partial x_{3}} \\
\frac{\partial f_{2}}{\partial x_{1}} & \frac{\partial f_{2}}{\partial x_{2}} & \frac{\partial f_{2}}{\partial x_{3}} \\
\frac{\partial f_{3}}{\partial x_{1}} & \frac{\partial f_{3}}{\partial x_{2}} & \frac{\partial f_{3}}{\partial x_{3}}
\end{array}\right]_{\substack{x_{1}=x_{10} \\
x_{2}=x_{0} \\
x_{3}=x_{30}}}^{\mathbf{g}(x)=\mathbf{f}(\mathbf{x}, \mu)-\mathbf{A x} .},
\end{aligned}
$$

A linear transformation is performed on the three-dimensional system: $\mathbf{x}=\mathbf{P y}+\mathbf{x}_{0}\left(\mu_{0}\right)$, where the first two columns of $\mathbf{P}=\left[\operatorname{Re}\left(\lambda_{1}\right) \operatorname{Im}\left(\lambda_{1}\right) \lambda_{3}\right]$ are the real and imaginary parts of the eigenvector corresponding to the eigenvalue $\lambda_{1}=i \omega_{0}(\mu)$ of the Jacobian matrix $\mathbf{A}$, and the third column is the eigenvector corresponding to the real eigenvalue $\lambda_{3}$. It can be seen from formulae (19) and (20) that $\dot{y}=\mathbf{J y}+\mathbf{P}^{-1} \mathbf{f}\left(\mathbf{P y}+\mathbf{x}_{0}\left(\mu_{0}\right)\right)-\mathbf{J y}=\mathbf{J y}+\mathbf{F}$, where $\mathbf{J}=\mathbf{P}^{-1} \mathbf{A P}$ is the Jordan matrix of the system and $\mathbf{F}$ is the nonlinear term: $\mathbf{F}=\mathbf{P}^{-1} \mathbf{f}\left(\mathbf{P y}+\mathbf{x}_{0}\left(\mu_{0}\right)\right)-\mathbf{J y}$. Its component form is as follows:

$$
\left[\begin{array}{c}
F_{1} \\
F_{2} \\
F_{3}
\end{array}\right]=\mathbf{P}^{-1}\left[\begin{array}{l}
f_{1}\left(\mathbf{P y}+\mathbf{x}_{0}\left(\mu_{0}\right)\right) \\
f_{2}\left(\mathbf{P y}+\mathbf{x}_{0}\left(\mu_{0}\right)\right) \\
f_{3}\left(\mathbf{P y}+\mathbf{x}_{0}\left(\mu_{0}\right)\right)
\end{array}\right]-\mathbf{J}\left[\begin{array}{c}
y_{1} \\
y_{2} \\
y_{3}
\end{array}\right] .
$$

The basic formula for determining the Hopf bifurcation type of a three-dimensional nonlinear system is still formula (22), which is obtained from formula (24), when $n=3$ as follows:

$$
g_{21}=G_{21}+2 G_{110}^{1} w_{11}^{1}+G_{101}^{1} w_{20}^{1} \text {. }
$$

Therefore,

$$
\beta_{2}=\operatorname{Re}\left\{\frac{g_{20} g_{11}}{\omega_{0}} i+G_{21}\right\}+\operatorname{Re}\left\{2 G_{110}^{1} w_{11}^{1}+G_{101}^{1} w_{20}^{1}\right\},
$$

where 


$$
\begin{aligned}
G_{110}^{1}= & \frac{1}{2}\left(\frac{\partial^{2} F_{1}}{\partial y_{1} \partial y_{3}}+\frac{\partial^{2} F_{2}}{\partial y_{2} \partial y_{3}}+i\left(\frac{\partial^{2} F_{2}}{\partial y_{1} \partial y_{3}}-\frac{\partial^{2} F_{1}}{\partial y_{2} \partial y_{3}}\right)\right), \\
G_{101}^{1}= & \frac{1}{2}\left(\frac{\partial^{2} F_{1}}{\partial y_{1} \partial y_{3}}-\frac{\partial^{2} F_{2}}{\partial y_{2} \partial y_{3}}+i\left(\frac{\partial^{2} F_{2}}{\partial y_{1} \partial y_{3}}+\frac{\partial^{2} F_{1}}{\partial y_{2} \partial y_{3}}\right)\right), \\
w_{11}^{1}= & -\frac{1}{4 \lambda_{3}}\left(\frac{\partial^{2} F_{3}}{\partial y_{1}^{2}}+\frac{\partial^{2} F_{3}}{\partial y_{2}^{2}}\right), \\
w_{20}^{1}= & -\frac{1}{4\left(\lambda_{3}^{2}+4 \omega_{0}^{2}\right)}\left[\lambda_{3}\left(\frac{\partial^{2} F_{3}}{\partial y_{1}^{2}}-\frac{\partial^{2} F_{3}}{\partial y_{2}^{2}}\right)+4 \omega_{0} \frac{\partial^{2} F_{3}}{\partial y_{1} \partial y_{2}}\right] \\
& -i \frac{1}{2\left(\lambda_{3}^{2}+4 \omega_{0}^{2}\right)}\left[\omega_{0}\left(\frac{\partial^{2} F_{3}}{\partial y_{1}^{2}}-\frac{\partial^{2} F_{3}}{\partial y_{2}^{2}}\right)-\lambda_{3} \frac{\partial^{2} F_{3}}{\partial y_{1} \partial y_{2}}\right] .
\end{aligned}
$$

$\lambda_{3}$ is the real eigenvalue at the equilibrium point, so

$$
\begin{aligned}
\operatorname{Re}\left(2 G_{110}^{1} w_{11}^{1}+G_{101}^{1} w_{20}^{1}\right)= & -\frac{1}{4 \lambda_{3}}\left[\left(\frac{\partial^{2} F_{2}}{\partial y_{2} \cdot \partial y_{3}}+\frac{\partial^{2} F_{1}}{\partial y_{1} \cdot \partial y_{3}}\right) \cdot\left(\frac{\partial^{2} F_{3}}{\partial y_{2}^{2}}+\frac{\partial^{2} F_{3}}{\partial y_{1}^{2}}\right)\right] \\
& +\frac{\omega_{0}}{4\left(\lambda_{3}^{2}+4 \omega_{0}^{2}\right)}\left[\left(\frac{\partial^{2} F_{1}}{\partial y_{2} \cdot \partial y_{3}}+\frac{\partial^{2} F_{2}}{\partial y_{1} \cdot \partial y_{3}}\right) \cdot\left(\frac{\partial^{2} F_{3}}{\partial y_{1}^{2}}-\frac{\partial^{2} F_{3}}{\partial y_{2}^{2}}\right)\right] \\
& -\frac{\lambda_{3}}{4\left(\lambda_{3}^{2}+4 \omega_{0}^{2}\right)}\left[\frac{\partial^{2} F_{3}}{\partial y_{1} \cdot \partial y_{2}}\left(\frac{\partial^{2} F_{1}}{\partial y_{2} \cdot \partial y_{3}}+\frac{\partial^{2} F_{2}}{\partial y_{1} \cdot \partial y_{3}}\right)\right] \\
& -\frac{\lambda_{3}}{8\left(\lambda_{3}^{2}+4 \omega_{0}^{2}\right)}\left[\left(\frac{\partial^{2} F_{1}}{\partial y_{1} \cdot \partial y_{3}}-\frac{\partial^{2} F_{2}}{\partial y_{2} \cdot \partial y_{3}}\right) \cdot\left(\frac{\partial^{2} F_{3}}{\partial y_{1}^{2}}-\frac{\partial^{2} F_{3}}{\partial y_{2}^{2}}\right)\right] \\
& -\frac{\omega_{0}}{2\left(\lambda_{3}^{2}+4 \omega_{0}^{2}\right)}\left[\frac{\partial^{2} F_{3}}{\partial y_{1} \cdot \partial y_{2}}\left(\frac{\partial^{2} F_{1}}{\partial y_{1} \cdot \partial y_{3}}-\frac{\partial^{2} F_{2}}{\partial y_{2} \cdot \partial y_{3}}\right)\right] .
\end{aligned}
$$

It is known in Section 4 that

$$
\begin{aligned}
\operatorname{Re}\left\{\frac{g_{20} g_{11}}{\omega_{0}} i+G_{21}\right\}= & \frac{1}{8 \omega_{0}}\left[\frac{\partial^{2} F_{1}}{\partial y_{2}^{2}} \cdot \frac{\partial^{2} F_{2}}{\partial y_{2}^{2}}-\frac{\partial^{2} F_{1}}{\partial y_{1}^{2}} \cdot \frac{\partial^{2} F_{2}}{\partial y_{1}^{2}}+\frac{\partial^{2} F_{1}}{\partial y_{1} \cdot \partial y_{2}} \cdot\left(\frac{\partial^{2} F_{1}}{\partial y_{1}^{2}}+\frac{\partial^{2} F_{1}}{\partial y_{2}^{2}}\right)-\frac{\partial^{2} F_{2}}{\partial y_{1} \cdot \partial y_{2}}\left(\frac{\partial^{2} F_{2}}{\partial y_{1}^{2}}+\frac{\partial^{2} F_{2}}{\partial y_{2}^{2}}\right)\right] \\
& \frac{1}{8}\left[\frac{\partial^{3} F_{2}}{\partial y_{2}^{3}}+\frac{\partial^{3} F_{1}}{\partial y_{1} \cdot \partial y_{2}^{2}}+\frac{\partial^{3} F_{2}}{\partial y_{1}^{2} \cdot \partial y_{2}}+\frac{\partial^{3} F_{1}}{\partial y_{1}^{3}}\right] .
\end{aligned}
$$

Therefore, the determination formula of the Hopf bifurcation type of the three-dimensional Prescott model is as follows: 


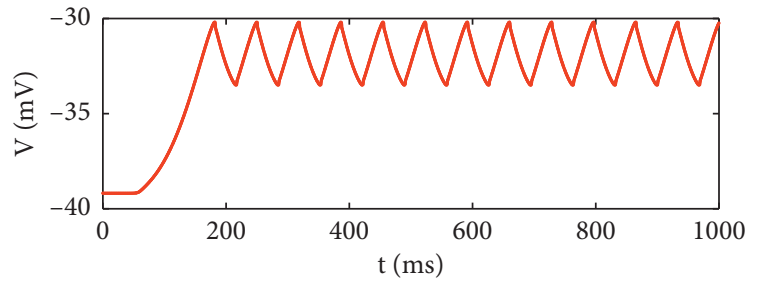

(a)

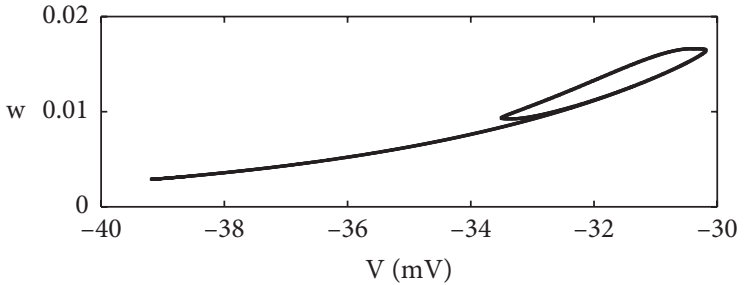

(b)

FIgURE 4: The firing response curve (a) and its phase trajectory (b) in the neighborhood of the supercritical Hopf bifurcation after adding the controller in the Prescott model.

$$
\begin{aligned}
\beta_{2}= & \frac{1}{8 \omega_{0}}\left[\frac{\partial^{2} F_{1}}{\partial y_{2}^{2}} \cdot \frac{\partial^{2} F_{2}}{\partial y_{2}^{2}}-\frac{\partial^{2} F_{1}}{\partial y_{1}^{2}} \cdot \frac{\partial^{2} F_{2}}{\partial y_{1}^{2}}+\frac{\partial^{2} F_{1}}{\partial y_{1} \cdot \partial y_{2}} \cdot\left(\frac{\partial^{2} F_{1}}{\partial y_{1}^{2}}+\frac{\partial^{2} F_{1}}{\partial y_{2}^{2}}\right)-\frac{\partial^{2} F_{2}}{\partial y_{1} \cdot \partial y_{2}}\left(\frac{\partial^{2} F_{2}}{\partial y_{1}^{2}}+\frac{\partial^{2} F_{2}}{\partial y_{2}^{2}}\right)\right] \\
& +\frac{1}{8}\left[\frac{\partial^{3} F_{2}}{\partial y_{2}^{3}}+\frac{\partial^{3} F_{1}}{\partial y_{1} \cdot \partial y_{2}^{2}}+\frac{\partial^{3} F_{2}}{\partial y_{1}^{2} \cdot \partial y_{2}}+\frac{\partial^{3} F_{1}}{\partial y_{1}^{3}}\right]-\frac{1}{4 \lambda_{3}}\left[\left(\frac{\partial^{2} F_{2}}{\partial y_{2} \cdot \partial y_{3}}+\frac{\partial^{2} F_{1}}{\partial y_{1} \cdot \partial y_{3}}\right) \cdot\left(\frac{\partial^{2} F_{3}}{\partial y_{2}^{2}}+\frac{\partial^{2} F_{3}}{\partial y_{1}^{2}}\right)\right] \\
& +\frac{\omega_{0}}{4\left(\lambda_{3}^{2}+4 \omega_{0}^{2}\right)}\left[\left(\frac{\partial^{2} F_{1}}{\partial y_{2} \cdot \partial y_{3}}+\frac{\partial^{2} F_{2}}{\partial y_{1} \cdot \partial y_{3}}\right) \cdot\left(\frac{\partial^{2} F_{3}}{\partial y_{1}^{2}}-\frac{\partial^{2} F_{3}}{\partial y_{2}^{2}}\right)\right]-\frac{\lambda_{3}}{4\left(\lambda_{3}^{2}+4 \omega_{0}^{2}\right)}\left[\frac{\partial^{2} F_{3}}{\partial y_{1} \cdot \partial y_{2}}\left(\frac{\partial^{2} F_{1}}{\partial y_{2} \cdot \partial y_{3}}+\frac{\partial^{2} F_{2}}{\partial y_{1} \cdot \partial y_{3}}\right)\right] \\
& -\frac{\lambda_{3}}{8\left(\lambda_{3}^{2}+4 \omega_{0}^{2}\right)}\left[\left(\frac{\partial^{2} F_{1}}{\partial y_{1} \cdot \partial y_{3}}-\frac{\partial^{2} F_{2}}{\partial y_{2} \cdot \partial y_{3}}\right) \cdot\left(\frac{\partial^{2} F_{3}}{\partial y_{1}^{2}}-\frac{\partial^{2} F_{3}}{\partial y_{2}^{2}}\right)\right]-\frac{\omega_{0}}{2\left(\lambda_{3}^{2}+4 \omega_{0}^{2}\right)}\left[\frac{\partial^{2} F_{3}}{\partial y_{1} \cdot \partial y_{2}}\left(\frac{\partial^{2} F_{1}}{\partial y_{1} \cdot \partial y_{3}}-\frac{\partial^{2} F_{2}}{\partial y_{2} \cdot \partial y_{3}}\right)\right] .
\end{aligned}
$$

From formula (29), it can be seen that after the twowhere dimensional Prescott model is added to the controller, its three-dimensional equation can be rewritten as follows:

$$
\begin{aligned}
\dot{V} & =f_{1}(V, w, z), \\
\dot{w} & =f_{2}(V, w, z), \\
\dot{z} & =f_{3}(V, w, z),
\end{aligned}
$$

$$
\begin{aligned}
& f_{1}=\frac{\left[I-g_{L}\left(V-E_{L}\right)-g_{\mathrm{Na}} m_{\infty}(V)\left(V-E_{\mathrm{Na}}\right)-g_{\mathrm{K}} w\left(V-E_{\mathrm{K}}\right)+u\right]}{c_{m}}, \\
& f_{2}=\frac{\varphi_{w}\left(w_{\infty}(V)-w\right)}{\tau_{w}(V)}, \\
& f_{3}=V-\xi z .
\end{aligned}
$$

$\mathbf{f}\left(\mathbf{P y}+\mathbf{x}_{0}\left(\mu_{0}\right)\right)$ in the nonlinear term can be deduced $\left(\mathbf{x}_{0}=\left(V_{0}, w_{0}, z_{0}\right), \mu_{0}=I_{0}\right)$ : 


$$
\begin{aligned}
f_{1}= & \frac{1}{c_{m}}\left[I_{0}-g_{L}\left(-E_{L}+V_{0}+P_{11} y_{1}+P_{12} y_{2}+P_{13} y_{3}\right)\right. \\
& -g_{\mathrm{K}}\left(-E_{\mathrm{K}}+V_{0}+P_{11} y_{1}+P_{12} y_{2}+P_{13} y_{3}\right) \cdot\left(w_{0}+P_{21} y_{1}+P_{22} y_{2}+P_{23} y_{3}\right) \\
& -0.5 g_{\mathrm{Na}}\left(-E_{\mathrm{Na}}+V_{0}+P_{11} y_{1}+P_{12} y_{2}+P_{13} y_{3}\right)\left(1+\tanh \left(\frac{V_{0}+P_{11} y_{1}+P_{12} y_{2}+P_{13} y_{3}-\beta_{m}}{\gamma_{m}}\right)\right) \\
& \left.+k\left(V_{0}+P_{11} y_{1}+P_{12} y_{2}+P_{13} y_{3}-\xi\left(z_{0}+P_{31} y_{1}+P_{32} y_{2}+P_{33} y_{3}\right)\right)^{3}\right], \\
f_{2}= & \cosh \left(\frac{V_{0}+P_{11} y_{1}+P_{12} y_{2}+P_{13} y_{3}-\beta_{w}}{2}\right) \\
& \cdot \varphi_{w}\left[-\left(w_{0}+P_{21} y_{1}+P_{22} y_{2}+P_{23} y_{3}\right)+0.5\left(1+\tanh \left(\frac{V_{0}+P_{11} y_{1}+P_{12} y_{2}+P_{13} y_{3}-\beta_{w}}{\gamma_{w}}\right)\right)\right], \\
f_{3}= & V_{0}+P_{11} y_{1}+P_{12} y_{2}+P_{13} y_{3}-\xi\left(z_{0}+P_{31} y_{1}+P_{32} y_{2}+P_{33} y_{3}\right) .
\end{aligned}
$$

Therefore, $\mathbf{F}=\mathbf{P}^{-1} \mathbf{f}\left(\mathbf{P y}+\mathbf{x}_{0}\left(\mu_{0}\right)\right)$ can be derived from above, and $\beta_{2}$ can be obtained using formula (37). Then, the type of Hopf bifurcation after adding the controller can be judged by $\beta_{2}$.

Since the Washout filter does not change the equilibrium point of the original system, the bifurcation parameter is $I_{0}=33.1813 \mu \mathrm{A} / \mathrm{cm}^{2}$, and the equilibrium point is $\left(V_{0}, w_{0}, z_{0}\right)=(-39.1842,0.002961,-78.3684)$. The eigenvalues at the bifurcation equilibrium point are $\lambda_{1}=-0.5$, $\lambda_{2}=0.07075 i$, and $\lambda_{3}=-0.07075 i$. The matrix $\mathbf{P}$ composed of the eigenvectors corresponding to the eigenvalues of the linearization matrix of the system is as follows:

$$
\mathbf{P}=\left[\begin{array}{ccc}
0 & 1 & 0 \\
0.10825087 e-3 & 0.55926765 e-3 & 0 \\
0.25884715 & 1.96591828 & 1
\end{array}\right]
$$

Therefore, $\mathbf{P}^{-1}$ can be derived and the nonlinear term $\mathbf{F}=\left[F_{1}(y), F_{2}(y), F_{3}(y)\right]^{T}$ of the governing equation can be obtained. $\beta_{2}=-0.00478458 k-0.026219655$ is calculated using formula (37). If the system is to produce a supercritical Hopf bifurcation here, $\beta_{2}<0$ is required. So, when $k<-5.48$, the neuron model will have a supercritical Hopf at $I_{0}=33.1813 \mu \mathrm{A} / \mathrm{cm}^{2}$.

The membrane voltage curve and phase trajectory of the model at the Hopf bifurcation after adding the controller are shown in Figure 4. The firing trajectory converges to a stable limit cycle. At this time, the system produces a small amplitude limit cycle attractor. It shows that after the controller is applied, the Hopf bifurcation type of the neuron model is changed from an unstable subcritical Hopf bifurcation to a stable supercritical Hopf bifurcation.

\section{Conclusions}

This paper uses analytical methods to study the bifurcation characteristics of the two-dimensional Prescott neuron model and uses numerical simulation to verify the corresponding conclusions. On the basis of the traditional Hopf bifurcation theory, adding the research method of the Prescott model, the analytical conditions for Hopf bifurcation in the Prescott model are obtained. The analytical expression of the determination formula $\beta_{2}$ for the Hopf bifurcation type of the Prescott model is derived, and the Hopf bifurcation type of the model is judged according to $\beta_{2}$. By applying a Washout filter to the Prescott model, the Hopf bifurcation type of the model is changed, and the subcritical Hopf bifurcation is transformed into a supercritical Hopf bifurcation, thereby changing the firing characteristics of neurons. This can achieve the purpose of eliminating the hidden firing behavior of the system and further controlling the stable area of the neuronal system. The results obtained will help to study the pathogenesis of neuron-related diseases and hidden dynamic behavior, which is of great significance to the prevention and control of neuronal diseases.

\section{Data Availability}

The data used to support the findings of this study are available from the corresponding author upon request.

\section{Conflicts of Interest}

The authors declare that they have no conflicts of interest to this work.

\section{Acknowledgments}

This work was supported by the Scientific Research Fund of Liaoning Provincial Education Department (grant nos. LG202028 and LG201930).

\section{References}

[1] A. Jangid, L. Chaudhary, and K. Sharma, "Computational neuroscience and its applications: a review," in Intelligent Energy Management Technologies. Algorithms for Intelligent 
System, M. Shorif Uddin, A. Sharma, K. L. Agarwal, (Eds.), Springer, Singapore, 2021.

[2] E. M. Izhikevich, Dynamical Systems in Neuroscience: The Geometry of Excitability and Bursting, MIT Press, Cambridge, MA, USA, 2007.

[3] X. Li, C. Yuan, and B. Shan, "System identification of neural signal transmission based on back propagation neural network," Mathematical Problems in Engineering, vol. 2020, Article ID 9652678, 8 pages, 2020.

[4] A. V. M. Herz, T. Gollisch, C. K. Machens, and D. Jaeger, "Modeling single-neuron dynamics and computations: a balance of detail and abstraction," Science, vol. 314, no. 5796, pp. 80-85, 2006.

[5] Q. Qin, Y. Liu, B. Shan et al., "Spike-sorting analysis of neural electrical signals evoked by acupuncture based on model," Cognitive Neurodynamics, vol. 15, no. 3, pp. 1-10, 2020.

[6] Z. He and D. Fan, "A tunable magnetic skyrmion neuron cluster for energy efficient artificial neural network," in Proceedings of the Design, Automation \& Test in Europe Conference \& Exhibition, Lausanne, Switzerland, March 2017.

[7] O. V. Maslennikov, D. S. Shchapin, and V. I. Nekorkin, "Transient sequences in a hypernetwork generated by an adaptive network of spiking neurons," Philosophical Transactions of the Royal Society a Mathematical Physical and Engineering Sciences, vol. 375, no. 2096, 2017.

[8] Q. Jin, J. Wang, X. Wei et al., "Action potential initial dynamical control and analysis of a minimum neuron model," Acta Physica Sinica, vol. 60, no. 9, pp. 774-782, 2011.

[9] S. A. Prescott, Y. De Koninck, and T. J. Sejnowski, "Biophysical basis for three distinct dynamical mechanisms of action potential initiation," PLoS Computational Biology, vol. 4, no. 10, Article ID e1000198, 2008.

[10] O. A. Hafez and A. Gottschalk, "Altered neuronal excitability in a Hodgkin-Huxley model incorporating channelopathies of the delayed rectifier potassium channel," Journal of Computational Neuroscience, vol. 48, no. 4, pp. 377-386, 2020.

[11] R. Cai, Y. Liu, J. Duan et al., "State transitions in the MorrisLecar model under stable Lévy noise," The European Physical Journal B: Condensed Matter and Complex Systems, vol. 93, 2020.

[12] Y. Liu, W.-j. Xu, J. Ma, F. Alzahrani, and A. Hobiny, "A new photosensitive neuron model and its dynamics," Frontiers of Information Technology \& Electronic Engineering, vol. 21, no. 9, pp. 1387-1396, 2020.

[13] S. Chen, Y. Zou, and X. Zhang, "An efficient method for Hopf bifurcation control in fractional-order neuron model," IEEE Access, vol. 7, pp. 77490-77498, 2019.

[14] L. Gu, P. Gong, and H. Wang, "Hopf bifurcation and turing instability analysis for the gierer-meinhardt model of the depletion type," Discrete Dynamics in Nature and Society, vol. 2020, Article ID 5293748, 10 pages, 2020.

[15] J. Wang, B. Lu, S. Liu et al., "Bursting types and bifurcation analysis in the pre-btzinger complex respiratory rhythm neuron," International Journal of Bifurcation and Chaos, vol. 27, no. 1, Article ID 1750010, 2017.

[16] S. Shi, H. Wang, and M. Xiao, "PD control at the Hopf bifurcation point of a neuron system with inertia and delay," in Proceedings of the 3rd International Conference on Artificial Intelligence, Automation and Control Technologies, Xi'an, China, April 2019.

[17] L. Zhou, Z. Zhao, and F. Chen, "Stability and Hopf bifurcation analysis of a new four-dimensional hyper-chaotic system," Modern Physics Letters B, vol. 34, Article ID 2050327, 2020.
[18] B. Bao, L. Hou, Y. Zhu et al., "Bifurcation analysis and circuit implementation for a tabu learning neuron model," International Journal of Electronics and Communications, vol. 121, Article ID 153235, 2020.

[19] B. D. Hassard, N. D. Kazarinoff, and Y. H. Wan, Theory and Applications of Hopf Bifurcation, pp. 86-91, Cambridge University Press, Cambridge, UK, 1981. 